\title{
Ангармонические блоховские осцилляции электронов в электрически смещенных сверхрешетках
}

\author{
() К.А. Иванов ${ }^{+*}$, Е.И. Гиршова ${ }^{+*}$, М.А. Калитеевский ${ }^{+*}$, \\ S.J. Clark ${ }^{\vee}$, A.J. Gallant ${ }^{\bullet}$ \\ + Университет ИТМО, \\ 197101 Санкт-Петербург, Россия \\ * Санкт-Петербургский академический университет - \\ научно-образовательный центр нанотехнологий Российской академии наук, \\ 194021 Санкт-Петербург, Россия \\ ৯ Физико-технический институт им. А.Ф. Иоффре Российской академии наук, \\ 194021 Санкт-Петербург, Россия \\ ' Department of Physics, University of Durham, \\ Durham, DH1 3LE, UK \\ - School of Engineering and Computing Sciences, University of Durham, \\ Durham, DH1 3LE, UK \\ E-mail: kivanov@corp.ifmo.ru
}

(Получена 27 апреля 2016 г. Принята к печати 10 мая 2016 г.)

Рассмотрено явление ангармонических блоховских осцилляций, т.е. имеющих частоту, кратную блоховской. При помощи расчетов из первых принципов найдена зонная структура политипов карбида кремния, где наблюдались такие осцилляции. Для этих политипов построен одномерный модельный потенциал, позволяющий рассчитывать штарковские волновые функции в смещенной сверхрешетке. С использованием метода матриц переноса найдены энергетические уровни (так называемая штарковская лестница) и вычислены волновые функции электрона. Показано, что возможны излучательные переходы как между соседними штарковскими уровнями, на блоховской частоте, так и прыжки через несколько уровней, сопровождающиеся излучением на частотах, кратных блоховской. Рассчитанные вероятности эмиссии между уровнями штарковской лестницы растут вместе с ростом приложенного поля.

\section{1. Введение}

Приложение электрического поля $F$ к электрону в периодическом потенциале периодом $d$ приводит к тому, что его движение из поступательного становится колебательным [1,2], с частотой $\omega$, определяемой из выражения

$$
\hbar \omega=e F d
$$

где $e$ - заряд электрона.

В одномерном периодическом потенциале зависимость энергии электрона от его волнового вектора $K$ также периодическая. Ускоренный приложенным электрическим полем электрон по достижении энергии, соответствующей точке перегиба дисперсионной зависимости, имеет отрицательную эффективную массу, так что его групповая скорость падает с последующим ростом энергии. Когда электрон достигает границы зоны Бриллюэна, его групповая скорость меняет знак, и он начинает движение обратно. Такое колебательное движение, носящее название блоховских осцилляций (БО), требует, чтобы энергия электрона достигала соответствующей верхней границе энергетической зоны, что определяет пороговое значение электрического поля $F_{\text {th }}[3,4]$ :

$$
W=e F_{\mathrm{th}} l
$$

где $W$ - ширина зоны, $l-$ средняя длина свободного пробега электрона.
Колебательное движение электрона должно сопровождаться испусканием электромагнитного излучения с частотой, определенной в уравнении (1). Более точная картина БО может быть получена при следующем квантово-механическом рассмотрении. Приложение электрического поля расщепляет мини-зону на набор локализованных состояний (так называемая штарковских состояний), разделенных по энергии равными интервалами, данными в уравнении (1), т. е. происходит локализация Ванье-Штарка $[5,6]$. Электромагнитное излучение испускается вследствие переходов между уровнями в так называемой штарковской лестнице.

В большинстве естественных кристаллов наблюдение БО невозможно на практике в связи с большой шириной мини-зоны, что требует слишком сильных полей, превосходящих пределы прочности кристалла. В 1970 г. в основополагающей работе Esaki \& Tsu [7] были предложены искусственные сверхрешетки с минизонным спектром в качестве предполагаемой основы для разработки источников ТГц-излучения. Некоторые признаки БО, такие как отрицательная дифференциальная проводимость $[8,9]$, штарковская локализация $[10,11]$ были экспериментально продемонстрированы. При этом рассеяние электронов на интерфейсах не позволяет создать эффективные источники ТГц-излучения на основе эффекта БО в искусственных СР.

Карбид кремния представляет собой естественную сверхрешетку [12-14]. Кристаллическая решетка карби- 


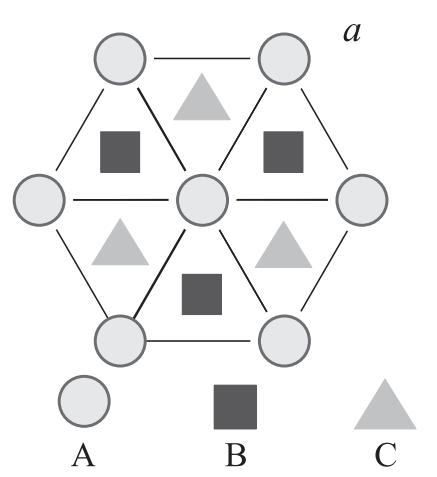

$c$

$(4 H)$
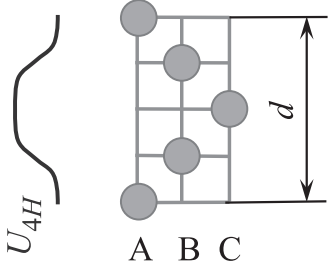

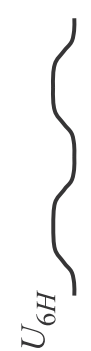

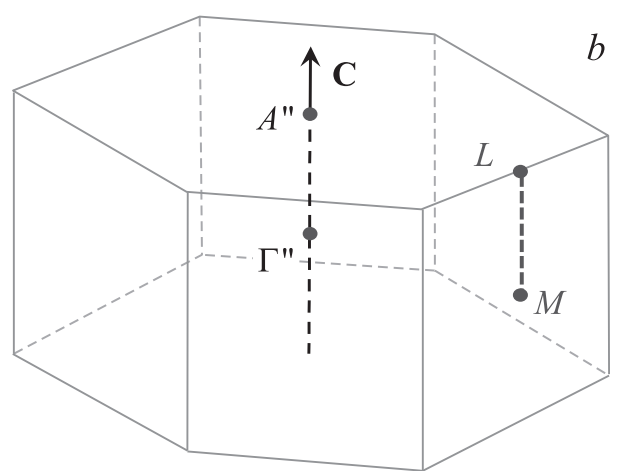

$b$

$(8 H)$

$e$
$(6 H)$

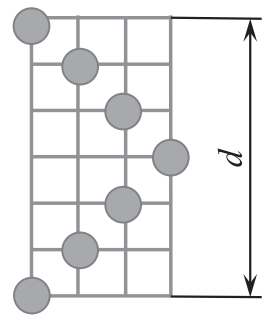

A B C A
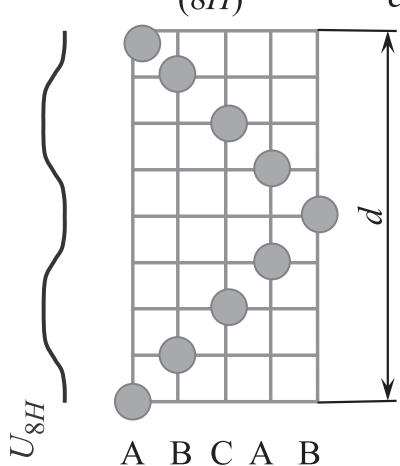

A B C A B

Рис. 1. $a-$ расположение атомов в плотноупакованных слоях. $b-$ зона Бриллюэна в гексагональной кристаллической решетке. Ось $C$ совпадает с осью естественной $\mathrm{CP}$ в $\mathrm{SiC}$. Взаимное положение атомных слоев (рамсделловский зигзаг) в $4 H-\mathrm{SiC}(c)$, $6 H$ - $\mathrm{SiC}(d)$ и $8 H-\mathrm{SiC}(e)$. Каждый круг обозначает пару атомов $\mathrm{Si}-\mathrm{C}$. Профиль модельного потенциала вдоль оси симметрии обозначен $U_{4 H}$ для $4 H-\mathrm{SiC}, U_{6 H}$ для $6 H-\mathrm{SiC}$ и $U_{8 H}$ для $8 H$-SiC. Период одномерного потенциала равен $d / 2$ для $U_{6 H}$ и $U_{8 H}$, в то время как для $U_{4 H}$ период одномерного потенциала соответствует $d$.

да кремния может быть рассмотрена подобно решеткам арсенида галлия или кремния, как набор плотно упакованных слоев. В отличие от упаковки типа ABCABC (как в структуре цинковой обманки) или ABAB (в вюрцитных структурах), политипы карбида кремния имеют более сложную структуру, например, в политипе $4 H$ слои чередуются по схеме $\mathrm{ABCB}$, как показано на рис. 1. Из-за наличия периода, кратного расстоянию между слоями, образуется естественная СР с минизонной энергетической структурой, как показано на рис. 2 и 3 . Недавно было продемонстрировано испускание ТГц-излучения мощностью более 10 мкВт смещенной структурой $6 \mathrm{H}-\mathrm{SiC}$ при температуре жидкого гелия [15].

В настоящее время считается, что БО являются гармоническими, что может сопровождаться излучением с единственной частотой, определяемой формулой (1). Квантово-механический анализ динамики электрона был представлен в работах Luban et al. $[17,18]$ в приближении ближайших соседей. В рамках этого подхода действительно остается ненулевым только дипольный матричный элемент для переходов между соседними уровнями. С другой стороны, пространственный вид потенциала, вызывающего локализацию Ванье-Штарка, далек от параболического, что может приводить к ан- гармоническим осцилляциям и появлению ненулевых матричных элементов переходов между несоседними уровнями.

Данная работа направлена на исследование возможности излучения на двойной и тройной блоховской частоте в сверхрешетках на примере политипа карбида кремния $6 H$-SiC.

\section{2. Результаты и обсуждение}

Свойства электрона в периодическом потенциале однозначно задаются его дисперсионной зависимостью, которая может быть рассчитана с помощью теории функционала плотности [19]. При этом большое значение играет выбор конкретного функционала. Известно, что использование локальных и полулокальных функционалов (LDA и GGA) ведет к ошибочным расчетам энергетических характеристик для многих материалов, в том числе и для карбида кремния. В работе [20] указаны более точные подходы, из которых для данной задачи наиболее подходящим оказался метод sX-LDA (гибридный функционал с экранированным обменным взаимодействием). Поскольку все уточненные методы требуют увеличения объемов вычислений, сетка в обратном пространстве не может быть слишком частой, и для 

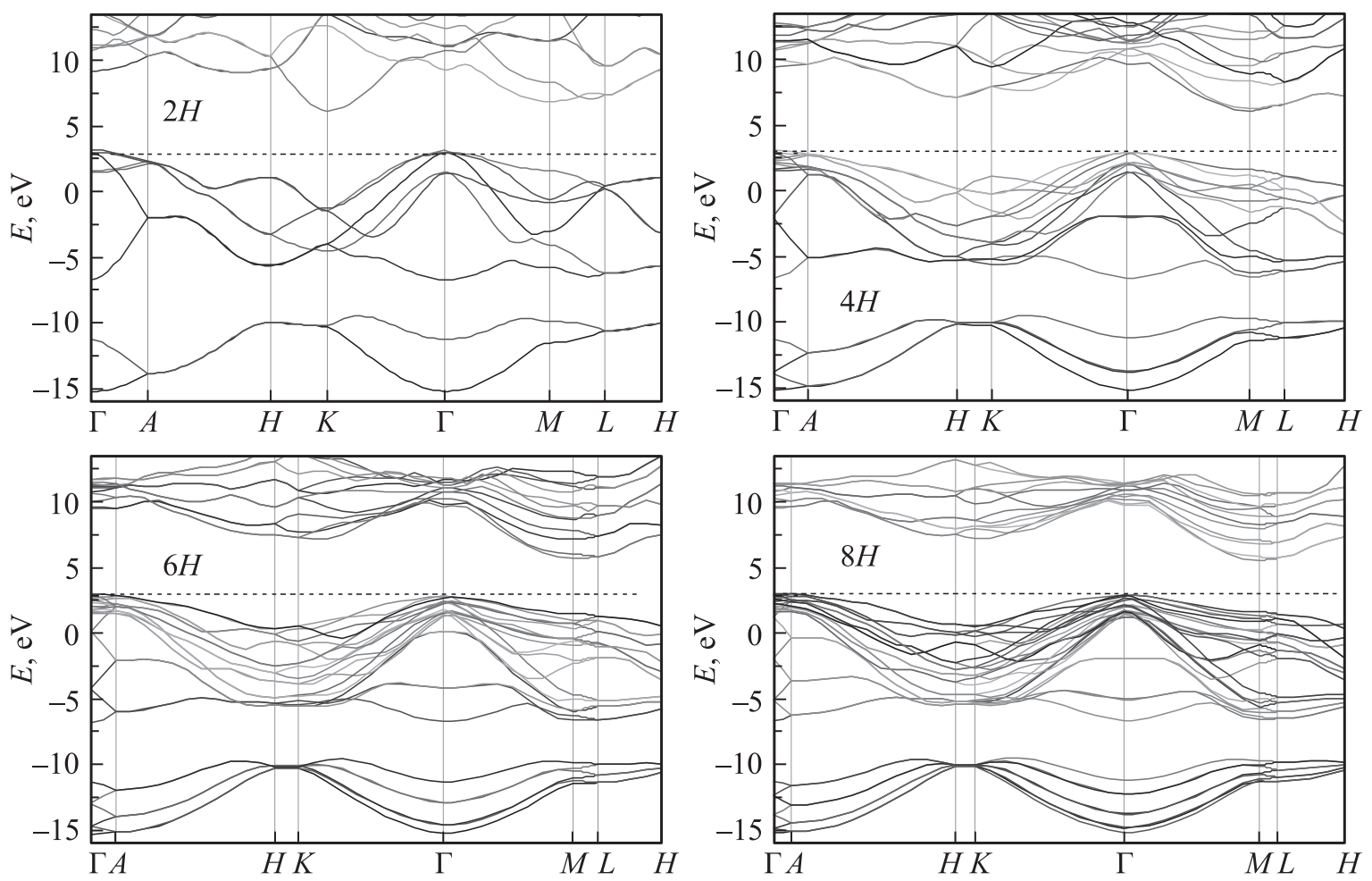

Рис. 2. Зонная структура политипов карбида кремния, вычисленная с использованием DFT-моделирования. Уровень Ферми обозначен штриховой линией.
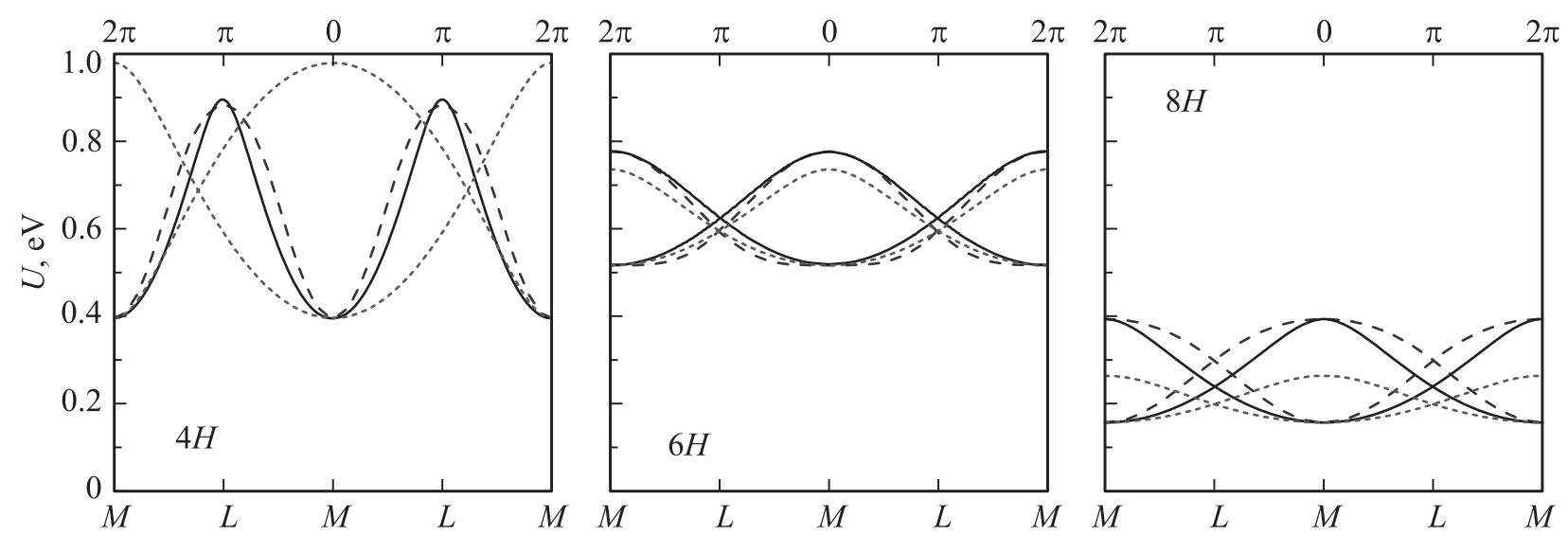

Рис. 3. Дисперсия вблизи дна зоны проводимости для политипов $\mathrm{SiC}$. Сплошная линия - дисперсия, соответствующая модельному потенциалу, длинные штрихи - расчет DFT-методом, короткие штрихи - дисперсия для потенциала из работы [18].

представленных расчетов ее размер составил $4 \times 4 \times 2$. На рис. 2 показаны зонные структуры политипов карбида кремния $2 H-\mathrm{SiC}, 4 H-\mathrm{SiC}, 6 H-\mathrm{SiC}$ и $8 H-\mathrm{SiC}$. Транспорт электронов в кристалле определяется участком дисперсионной зависимости, прилежащим к минимуму зоны проводимости. Блоховские осцилляции в $\mathrm{SiC}$ наблюдаются при приложении электрического поля вдоль оси симметрии кристалла (см. рис. 1), таким образом, для моделирования штарковских локализованных состояний достаточно рассмотреть участок дисперсионной зависимости $M L$. При приложении электрического поля периодические граничные условия, применяемые в тео- рии функционала плотности, становятся неприменимы, и расчеты из первых принципов становятся практически невозможны. Вместе с тем возможно описать сверхрешетку с помощью одномерного потенциала, в котором дисперсионная зависимость имеет такую же форму, как в случае расчета из первых принципов. Такое описание карбида кремния было впервые предложено в [18]. На рис. 3 показаны дисперсионные зависимости, полученные с помощью расчетов из первых принципов и с помощью одномерного модельного потенциала. Для сравнения показаны дисперсионные зависимости, предложенные в [18]. 
Гексагональные политипы $\mathrm{SiC}$ имеют зонную структуру, в которой минимум зоны проводимости в обратном пространстве располагается в точке симметрии $M$, и направление $M-L$ соответствует дну зоны проводимо-
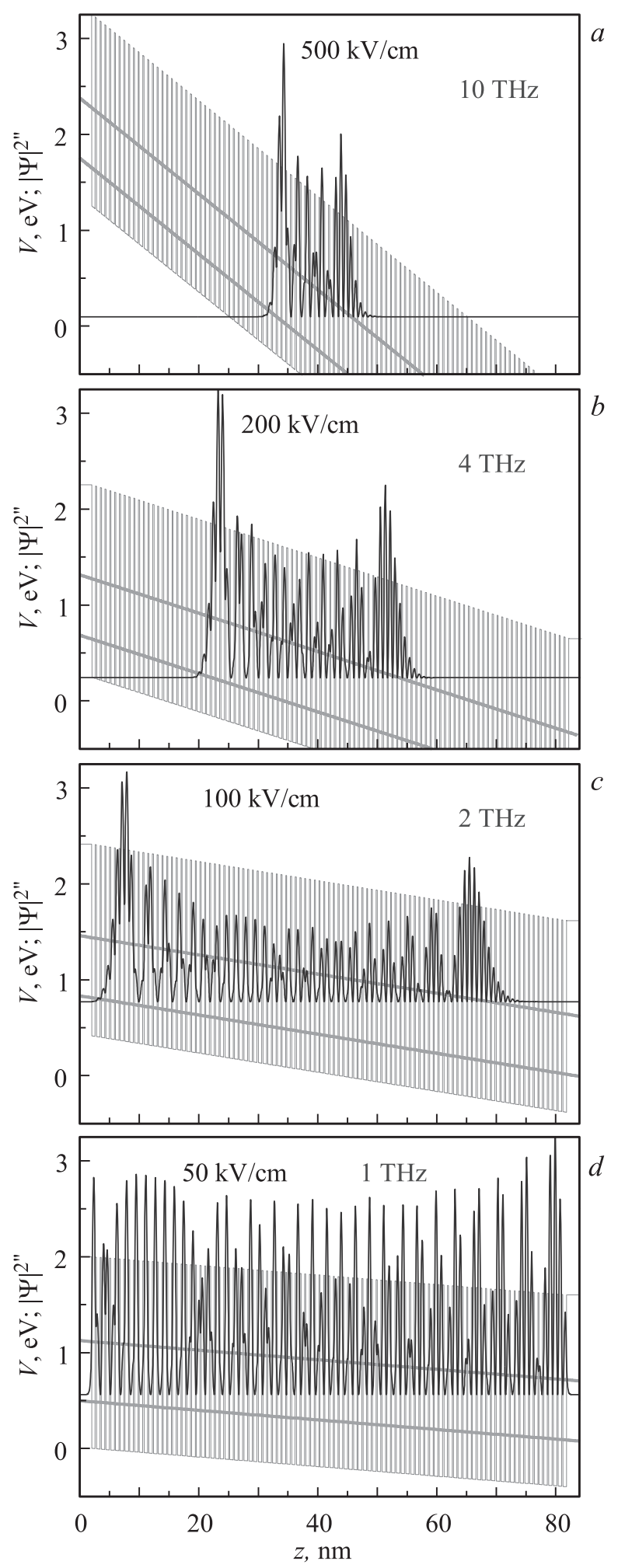

Рис. 4. Пространственное распределение плотности вероятности для локализованных состояний в сверхрешетке при смещениях $500(a), 200(b), 100(c), 50$ кВ/см $(d)$.
Параметры модельного потенциала для политипов карбида кремния

\begin{tabular}{l|l|l|l}
\hline \multicolumn{1}{c|}{ Политип } & $4 H$ & $6 H$ & $8 H$ \\
\hline Высота барьера, эВ & 1.9 & 2.35 & 0.79 \\
Ширина барьера, нм & 0.095 & 0.25 & 0.25 \\
Период потенциала (сумма ширины & 0.5 & 0.75 & 1.0 \\
барьера и ширины ямы), нм & & & \\
Модельная масса в барьере и яме, & 1.75 & 1.0 & 1.0 \\
масса свободного электрона & & &
\end{tabular}

сти. Следует отметить, что это направление соответствует движению электрона вдоль оси $c$ кристалла [21], и именно при движении электронов вдоль этого направления наблюдались БО. Для политипов $6 H, 8 H$ и более высоких порядков в точке $L$ возникает фолдинг дисперсионной зависимости, что видно на рис. 3 . Таким образом, в первой зоне Бриллюэна в интервале между $\bar{L}$ и $L$ (для $-\pi / d<K<\pi / d$ ) эффективная масса электрона все время положительна, и вышеописанного разворота электрона не происходит. Однако участок с отрицательной эффективной массой возникает во второй зоне Бриллюэна при $-2 \pi / d<K<-\pi / d$ и $\pi / d<K<2 \pi / d$. Вышеуказанное обстоятельство означает то, что в политипах $6 H$ и $8 H$ БО имеют место внутри второй зоны Бриллюэна. Это значит, что они характеризуются пространственным периодом, равным половине периода кристаллической решетки в направлении $c$. Таким образом, основные БО характеризуются частотой $\omega=e F d /(2 \hbar)$.

Получено, что наилучшее согласие „истинной“ дисперсионной зависимости и дисперсии в одномерном модельном потенциале получается при параметрах модельного потенциала, приведенных в таблице. Каждый период такого потенциала представляет собой прямоугольный барьер и соседствующую с ним прямоугольную яму. Отметим, что для $6 H$-SiC параметры модельного потенциала, приведенные в [18], схожи с параметрами, полученными в данной работе, а для политипов $4 \mathrm{H}-\mathrm{SiC}$ и $8 H$ - $\mathrm{SiC}$ имеют место существенные различия.

Одномерная сверхрешетка с точки зрения энергетической структуры представляет собой периодический потенциал. Приложенное электрическое поле преобразует потенциал в кусочно-линейный. Собственные значения энергии в таком потенциале удобно численно находить при помощи метода матриц переноса [22]. Каждому слою с постоянной энергией соответствуют две собственные функции $\exp \left( \pm i k_{i} x\right)$, где $k_{i}=\sqrt{2 m_{i}\left(E-U_{i}\right)} / \hbar-$ квазиволновой вектор в данном слое, $m_{i}$ - эффективная масса, $U_{i}-$ потенциальная энергия в слое. В базисе этих функций можно записать матрицу переноса через сам слой:

$$
\hat{M}_{i}=\left(\begin{array}{cc}
\exp \left(i k_{i} d_{i}\right) & 0 \\
0 & \exp \left(-i k_{i} d_{i}\right)
\end{array}\right)
$$




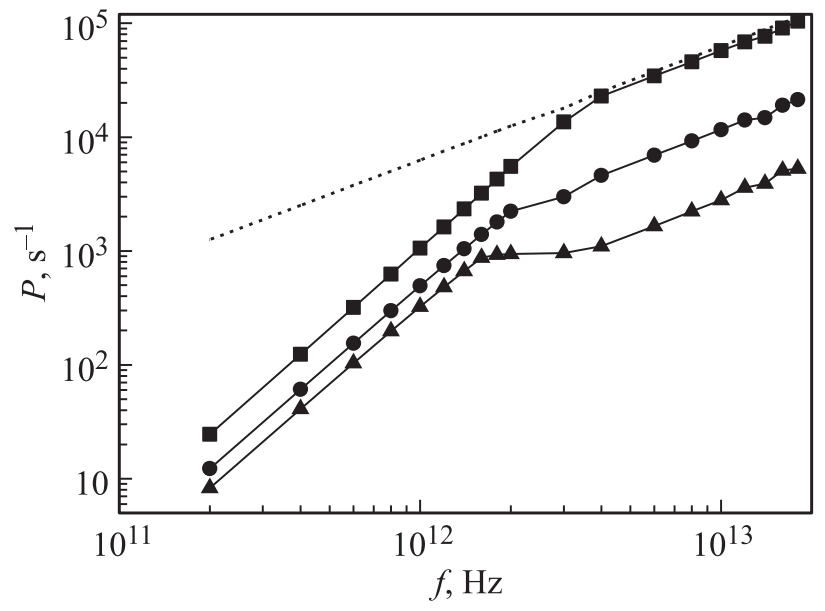

Рис. 5. Вероятность спонтанной эмиссии в зависимости от блоховской частоты для БО с одинарной (квадраты), двойной (круги) и тройной (треугольники) блоховскими частотами. Пунктирная линия отмечает аналитическую оценку по формуле (10). Отступление от теоретической оценки соответствует режиму малых полей, когда размер локализации штарковского состояния сравнивается с размером модельной структуры.

и через интерфейс между двумя слоями (пользуясь сохранением потока):

$$
\hat{T}_{i}=\frac{1}{2}\left(\begin{array}{cc}
1+\frac{m_{i+1} k_{i}}{m_{i} k_{i+1}} & 1-\frac{m_{i+1} k_{i}}{m_{i} k_{i+1}} \\
1-\frac{m_{i+1} k_{i}}{m_{i} k_{i+1}} & 1+\frac{m_{i+1} k_{i}}{m_{i} k_{i+1}}
\end{array}\right) .
$$

Матрица переноса $\hat{M}$ всей структуры есть произведение матриц переноса через все слои и все интерфейсы. Уравнение для собственных энергий получается из условия затухания волновой функции вне структуры:

$$
A\left(\begin{array}{l}
1 \\
0
\end{array}\right)=\hat{M}(E)\left(\begin{array}{l}
0 \\
1
\end{array}\right),
$$

где $A$ - произвольная постоянная. В итоге уравнение на собственные значения записывается в виде

$$
M_{22}(E)=0 .
$$

Волновые функции для каждого состояния получаются в результате склеивания отдельных экспонент, коэффициенты перед которыми также получаются при помощи матриц переноса.

Метод матриц переноса, примененный к периодической структуре с приложенным полем, дал штарковскую лестницу уровней, разделенных интервалами, равными блоховской энергии: $\left|E_{j+1}-E_{j}\right|=e F d$. На рис. 4 приведены плотности вероятности для рассчитанных локализованных штарковских состояний. При этом ха- рактеристический размер состояния равен

$$
s=W / e F,
$$

что соответствует пересечению мини-зоны линией энергии поля. За пределами этой длины состояние быстро затухает.

Для полей более 100 кВ/см штарковское состояние занимает только часть модельной структуры. С уменьшением поля его размер растет до тех пор, пока оно не выходит за пределы модельной структуры. В таком режиме локализация Ванье-Штарка сменяется локализацией внутри границ структуры.

Вероятность спонтанной эмиссии вычисляется при помощи золотого правила Ферми и определяется дипольным матричным элементом:

$$
P=\alpha\left|\left\langle\Psi_{l}|x| \Psi_{j}\right\rangle\right|^{2} \frac{n^{2} \omega^{3}}{\pi c^{2}},
$$

где $\alpha \approx 1 / 137$ - постоянная тонкой структуры, $n-$ показатель преломления, $\omega=\left(E_{l}-E_{j}\right) / \hbar$. Аналитическая оценка для $\left\langle\Psi_{l}|x| \Psi_{j}\right\rangle$ дает $[16,17]$

$$
\left|\left\langle\Psi_{l}|x| \Psi_{j}\right\rangle\right|=s / 4
$$

и, исходя из уравнений (1), (7), (8) и (9), можно оценить вероятность спонтанной эмиссии как

$$
P=\frac{a}{16 \pi}\left(\frac{n W d}{c \hbar}\right)^{2} \omega .
$$

На рис. 5 показана зависимость рассчитанных вероятностей спонтанной эмиссии от приложенного поля вместе с аналитической оценкой. Как видно, для частот выше 2 ТГц вероятность пропорциональна частоте, что соответствует оценке (6). Для меньших частот размер состояния превосходит размер модельной структуры, и матричный элемент практически перестает зависеть от приложенного поля. В таком режиме вероятность излучения пропорциональна плотности фотонных состояний, т. е. кубу частоты, согласно уравнению (8).

Видно также, что вероятности переходов, минуя один или два штарковских уровня - на двойной и тройной блоховской частоте, - имеют заметную величину, меньшую вероятности переходов на основной частоте на один и два порядка соответственно.

\section{3. Заключение}

Методом теории функционала плотности рассчитаны зоны политипов карбида кремния $2 H, 4 H, 6 H$ и $8 H$. Подобраны параметры одномерного модельного потенциала, в котором дисперсионная зависимость имеет вид практически идентичный участку дисперсионной зависимости политипов карбида кремния, ответственному за блоховские осцилляции. Показано, что блоховские осцилляции могут иметь ангармоническую природу: в 
сверхрешетках при приложении электрического поля возможно излучение на двойной и тройной блоховской частоте.

Работа поддержана грантом РНФ 16-12-10503 и грантом FP7 ITN NOTEDEV.

\section{Список литературы}

[1] F. Bloch. Z. Phys., 52, 555 (1928).

[2] C. Zener. Proc. Royal Soc. (London) Ser. A, 145, 523 (1934).

[3] K. Leo. Semicond. Sci. Technol., 13, 249 (1998).

[4] P. Voisin. Ann. Phys. (Fr.), 22, 681 (1997).

[5] G.H. Wannier. Phys. Rev., 117, 432 (1960).

[6] H.M. James. Phys. Rev., 76, 1611 (1949).

[7] L. Esaki, R. Tsu. IBM J. Res. Dev., 14, 61 (1970).

[8] A. Sibille, J.F. Palmier, H. Wong, J.C. Esanault, F. Mollot. Phys. Rev. Lett., 64, 52 (1990).

[9] F. Beltram et al. Phys. Rev. Lett., 64, 3167 (1990).

[10] P. Voisin et al. Phys. Rev. Lett., 61, 1639 (1988).

[11] E.E. Mendez, F. Agullo-Rueda, J.M. Hong. Phys. Rev. Lett., 60, 2426 (1988).

[12] L.S. Ramsdell. American Mineralogist, 32, 64 (1947).

[13] A.R. Verma, P. Krishna. Polymorphism and Polytypism in Crystals (Wiley, N. Y., 1966).

[14] V.I. Sankin, I.A. Stolichnov. Superlat. Microstr., 23, 999 (1998).

[15] V.I. Sankin, A.V. Andrianov, A.G. Petrov, A.O. Zakhar'in. Appl. Phys. Lett., 100, 111109 (2012).

[16] M. Luban. J. Math. Phys., 26, 2386 (1985).

[17] A.M. Bouchard, M. Luban. Phys. Rev. B, 52, 5105 (1995).

[18] Г.Б. Дубровский, А.А. Лепнева. ФТП, 19, 1252 (1977).

[19] S.J. Clark et al. Zeitschr. fuer Kristallographie, 220 (5-6), 567 (2005).

[20] P.J. Hasnip et al. Phil. Trans. Royal Soc. A, 372, 20130270 (2014).

[21] A. Laref, S. Laref. Phys. Status Solidi B, 245, 89 (2008).

[22] M.A. Kaliteevski, D.M. Beggs, S. Brand, R.A. Abram, V.V. Nikolaev. Phys. Rev. B, 73, 033106 (2006).

Редактор Г.А. Оганесян

\section{Anharmonic Bloch oscillations of electrons in electrically biased superlattices}

\author{
K.A. Ivanov ${ }^{+*}$, E.I. Girshova ${ }^{+*}$, M.A. Kaliteevski ${ }^{+* \Delta}$, \\ S.J. Clark ${ }^{\boldsymbol{1}}$, A.J. Gallant ${ }^{\bullet}$ \\ + ITMO University, \\ 197101 St. Petersburg, Russia \\ * St-Petersburg Academic University, \\ 194021 St. Petersburg, Russia. \\ $\triangle$ loffe Institute, \\ 194021 St. Petersburg, Russia \\ $\checkmark$ Department of Physics, University of Durham, \\ Durham, DH1 3LE, UK \\ - School of Engineering and Computing Sciences, \\ University of Durham, \\ Durham, DH1 3LE, UK
}

\begin{abstract}
The aim of the paper is to study the phenomena of anharmonic Bloch oscillation, i.e. those having multiple Bloch frequencies. The band structure of silicon carbide polymorphs where such oscillations occur was calculated ab initio. A model potential allowing calculation of optical effects is provided for these polymorphs. Eigen states (so called Stark states) with their corresponding wavefunctions were obtained using transfer matrix technique. It is shown that there is a possibility for optical transitions not only to adjacent Stark states, which accounts for single Bloch frequency radiation, but also to further states with multiple frequency radiation emission. The probability of these transitions grow with applied field increase.
\end{abstract}

